Published in final edited form as:

Nat Chem Biol. 2013 February ; 9(2): 69-70. doi:10.1038/nchembio.1161.

\title{
REDOX CONTROL:
}

\section{A black hole for oxidized glutathione}

\author{
Jakob R Winther and \\ Section for Biomolecular Sciences, Department of Biology, University of Copenhagen, \\ Copenhagen, Denmark
}

\author{
Ursula Jakob \\ Department of Molecular, Cellular and Developmental Biology, University of Michigan, Ann Arbor, \\ Michigan, USA, and the Department of Biological Chemistry, University of Michigan, Ann Arbor, \\ Michigan, USA
}

Ursula Jakob: ujakob@umich.edu

\begin{abstract}
There is a considerable amount of oxidized glutathione in living cells, yet it is virtually absent from the cytosol. The mystery of where it resides has now been solved. A study in baker's yeast revealed that oxidized glutathione is selectively stashed in vacuoles.
\end{abstract}

Reduced glutathione (GSH) and its oxidized counterpart, GSSG, serve as the major lowmolecular-weight redox buffer in eukaryotic cells. It was widely accepted that the overall cellular distribution between GSH and GSSG is about 20:1. Cytosols were typically considered to be more reducing, with a reported 'textbook' GSH:GSSG ratio of 100:1. This view, however, began crumbling with the introduction of GFP-based in vivo redox sensors, which reported much more extreme GSH:GSSG ratios on the order of 10,000:1 in the yeast cytosol. This result sparked some controversy in the field, in part because of the lack of a satisfactory explanation for where the missing GSSG could be. In this issue, Morgan and coworkers ${ }^{1}$ have now found the 'black hole of oxidized glutathione' by convincingly demonstrating that the yeast cell actively sequesters oxidized glutathione in its lysosomelike vacuole, hence maintaining a highly reducing environment even under extreme conditions of oxidative stress. This new finding suddenly places GSSG transport as a central player in cellular redox homeostasis and underpins the notion of very low GSSG in the cytosol.

Comprising three amino acids, of which the central one is cysteine, glutathione cycles between reduced and oxidized states, forming disulfide bonds with itself (GS-SG) or with protein thiols (PS-SG). Owing to its high abundance $(5-10 \mathrm{mM})$, glutathione has a pivotal role in keeping cytosolic proteins reduced and in protecting cells against reactive oxygen species. The reduced state of glutathione is maintained by the enzyme glutathione reductase, which in turn oxidizes the general cellular electron donor NADPH. It has long been clear that redox conditions in different compartments are maintained at different redox poise. For example, in the secretory pathway, proteins acquire structural disulfide bonds for increased stability, whereas in the cytosol, the activity of many enzymes depend on free cysteine

(c) 2013 Nature America, Inc. All rights reserved.

Competing financial interests

The authors declare no competing financial interests. 
thiols. On the basis of work from 1992, a GSH:GSSG ratio of 3:1 to 1:1 was estimated for the secretory pathway, whereas a ratio of 100:1 was proposed for the cytosol ${ }^{2}$.

This view began to change some ten years ago with the design of green fluorescent proteinbased redox sensors, which allowed for the first time direct measurements of the redox potential in vivo. The results surprised the redox world; the GSH:GSSG ratio was 10,000:1 and hence was two orders of magnitude higher than previously assumed ${ }^{3}$. Moreover, deletion of the gene encoding cytosolic glutathione reductase ( $G l r l)$ substantially increased whole-cell GSSG but only had a very minor impact on the cytosolic GSSG pool. There was good evidence that these sensors indeed measured the glutathione redox status, but with the low redox potential of the cytosol, virtually none of the GSSG could be accounted for. Where was the GSSG stash?

Morgan et al. ${ }^{1}$ have now elegantly resolved this enigma. By using a next-generation in vivo redox sensor, they tracked the whereabouts of GSSG in yeast strains where Glrl had been deleted. Guided by the hypothesis that GSSG might be sequestered in the yeast vacuole ${ }^{4}$, the functional equivalent of the mammalian lysosome, the authors asked what effect deletion of individual GSSG transporter candidates might have on total and cytosolic GSSG levels in cells lacking Glrl. They found that one member of the ABC family of ATP-dependent transporters encoded by the gene $Y c f l$ is responsible for the nearly quantitative export of oxidized GSSG to vacuoles (Fig. 1). This efficient transport almost completely alleviates any impact that the deletion of Glrl might otherwise have on the cytosolic redox potential. It is of note that cells lacking both glutathione reductase activity and the ability to export GSSG seem to engage a third GSSG removal strategy involving alternative members of the thioredoxin and glutaredoxin pathways. The mechanism (or mechanisms) by which these enzymes accomplish this task is, at this point, not completely clear.

The implications of the findings reported in this paper are intriguing. First and foremost, how do these observations in yeast translate to other cells, particularly mammalian cells, where GSSG is widely thought to be associated with the endoplasmic reticulum? As similar discrepancies in respect to the glutathione redox potential of the cytosol versus whole cells exist in mammalian cells ${ }^{5}$, the results reported in this study imply that mammalian cells have their own black hole for oxidized glutathione. And what happens to all of the GSSG that is sequestered in the vacuole? Can it be mobilized under starvation and used as an amino acid reserve, or does it have some other function? As every discovery sparks new questions, we are eagerly awaiting the next installment.

\section{References}

1. Morgan B, et al. Nat. Chem. Biol. 2013; 9:119-125. [PubMed: 23242256]

2. Hwang C, Sinskey AJ, Lodish HF. Science. 1992; 257:1496-1502. [PubMed: 1523409]

3. Østergaard H, Tachibana C, Winther JR. J. Cell Biol. 2004; 166:337-345. [PubMed: 15277542]

4. Lazard M, et al. FEBS J. 2011; 278:4112-4121. [PubMed: 21880115]

5. Dooley CT, et al. J. Biol. Chem. 2004; 279:22284-22293. [PubMed: 14985369] 


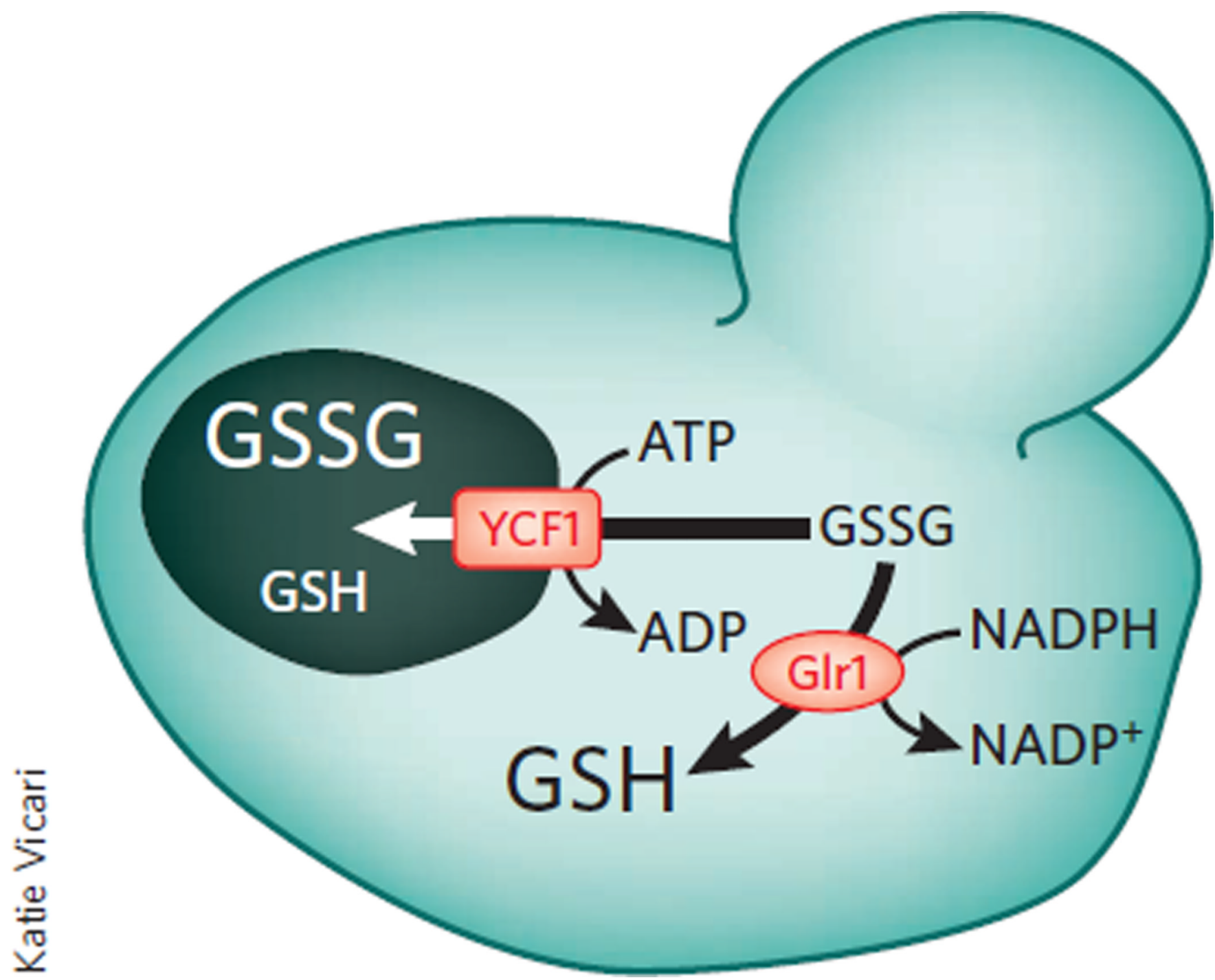

Figure 1.

A new star in redox homeostasis. In the cytosol, a high ratio between reduced (GSH) and oxidized (GSSG) glutathione is maintained by the enzyme glutaredoxin reductase (GIr1). In a competing reaction for GSSG removal, the membrane transporter YCF1 sweeps up GSSG for vacuolar sequestration. 